Supporting Information

\title{
Nitroxides as building blocks for nanoantioxidants
}

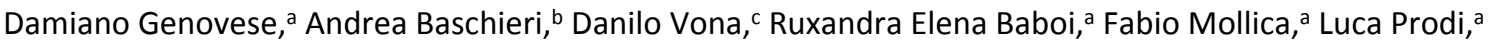
Riccardo Amorati*a and Nelsi Zaccheroni, ${ }^{a}$

a Department of Chemistry “G. Ciamician”, University of Bologna, via Selmi 3 and via San Giacomo 11, 40123, Bologna, Italy.

b Istituto per la Sintesi Organica e la Fotoreattività, Consiglio Nazionale delle Ricerche (ISOF - CNR), via Gobetti 101, 40129 Bologna, Italy.

c Department of Chemistry, University of Bari, via Orabona 4, I-70126 Bari, Italy

Content

Synthesis and FT-IR spectrum of silane-nitroxide $\mathbf{8}$

Absorption and emission spectra of PLUS-NO

TEM images (magnifications in the inset) of PluS-NO NPs

NMR and ESI mass spectra of compound 6

Numerical fitting of experimental X-band EPR spectra by Simlabel software

Numerical simulation of autoxidation of THF inhibited by nitroxides

References
Page

S2

S3

S3

S4 -S6

S7-S8

S9-S10

S10 
The silane nitroxide (8) was synthesized using the procedure already published in literature (ref S1). In a three-neck round-bottom flask 4-carboxy-TEMPO $(50 \mathrm{mg}, 0.25 \mathrm{mmol}$ ) was added to a solution of APTES (58 $\mu \mathrm{L}, 0.25 \mathrm{mmol}), \mathrm{EDC} \cdot \mathrm{HCl}(48 \mathrm{mg}, 0.25 \mathrm{mmol})$, and DMAP $(1.2 \mathrm{mg}, 0.025 \mathrm{mmol})$ in dry $\mathrm{CH}_{2} \mathrm{Cl}_{2}(10 \mathrm{~mL})$ at $0^{\circ} \mathrm{C}$ and the resulting mixture was stirred at room temperature for $48 \mathrm{~h}$. The reaction was monitored by TLC and GC-MS until the starting reagents had disappeared. The reaction mixture was quenched with water and extracted with $\mathrm{CH}_{2} \mathrm{Cl}_{2}(2 \times 50 \mathrm{~mL})$. The organic layers were combined, and the solvent was evaporated by a rotary evaporator. The pale orange oil residue was purified by column chromatography using ethyl acetate as the eluent to give an orange-colored oil (61\% yield).

MS (El, 70 eV) m/z (\%): 403 [M+], 373 (23), 357 (39), 327 (21), 271 (21), 202 (100), 160 (45), 124 (80), 107 (32), 79 (13). FTIR (KBr): v = 3320, 2970, 2920, $1650(\mathrm{C}=\mathrm{O}), 1550(\mathrm{NH}), 1450\left(\mathrm{CH}_{3}\right), 1380$ (NO)), 1325, 1102 (SiO), $1081 \mathrm{~cm}^{-1}$. Elemental analysis calc.d (\%) for $\mathrm{C}_{19} \mathrm{H}_{39} \mathrm{~N}_{2} \mathrm{O}_{5} \mathrm{Si}$ (403.6): C 56.54, H 9.74, N 6.94; found: C 56.79, H 9.85, N 7.05 .

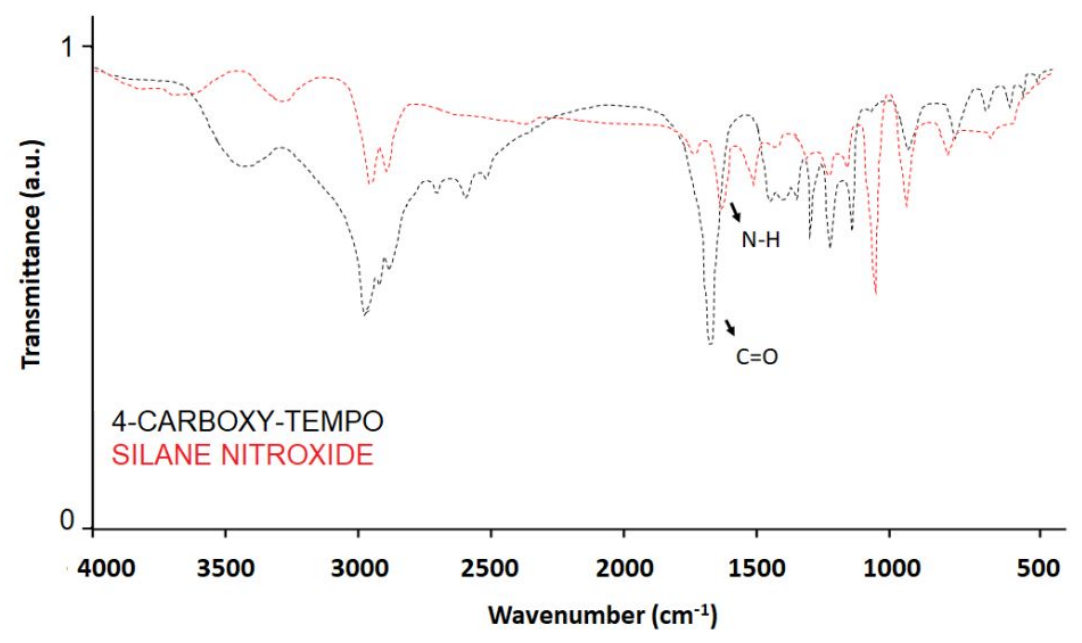

Figure S1. FT-IR spectrum of a KBr pellet of nitroxide 8 (silane nitroxide) and nitroxide 4 (4-carboxyTEMPO). 


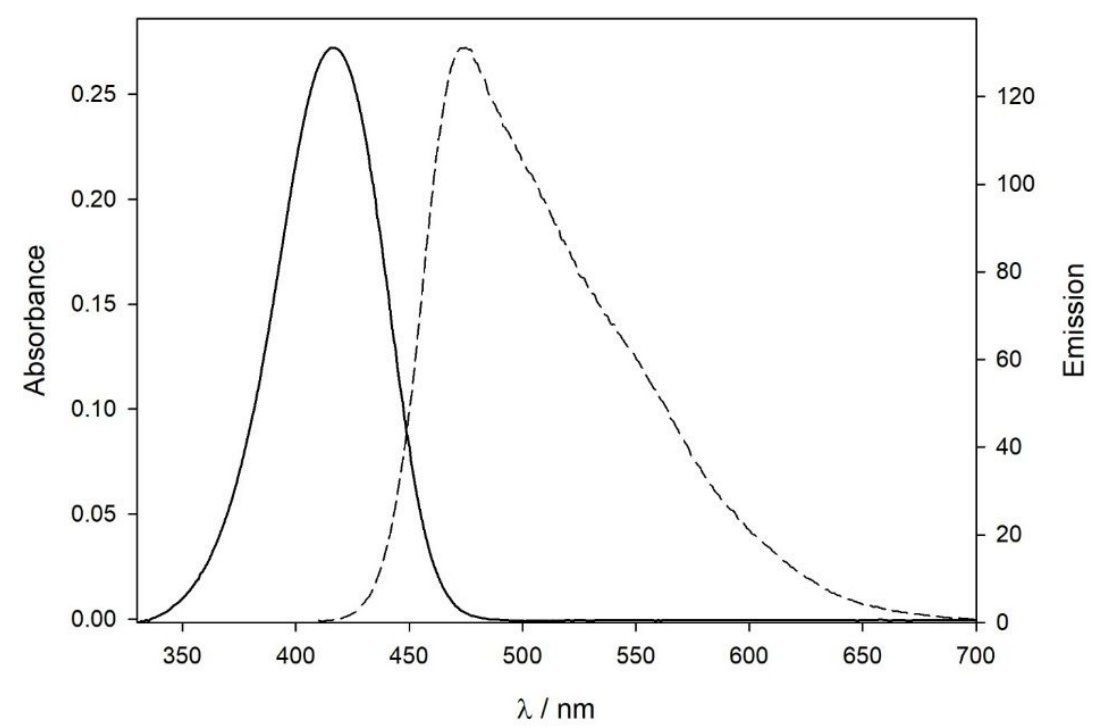

Figure S2. Absorption (continue line) and emission (dashed line, $\lambda_{\mathrm{ex}}=400 \mathrm{~nm}$ ) spectra of PluS-NO nanoparticles $\left(2.0 \times 10^{-7} \mathrm{M}\right.$ in $\mathrm{H}_{2} \mathrm{O}$ ). (Data obtained with a Perkin-Elmer Lambda 45 spectrophotometer and an Edinburgh FLS920 equipped with a photomultiplier Hamamatsu R928P)

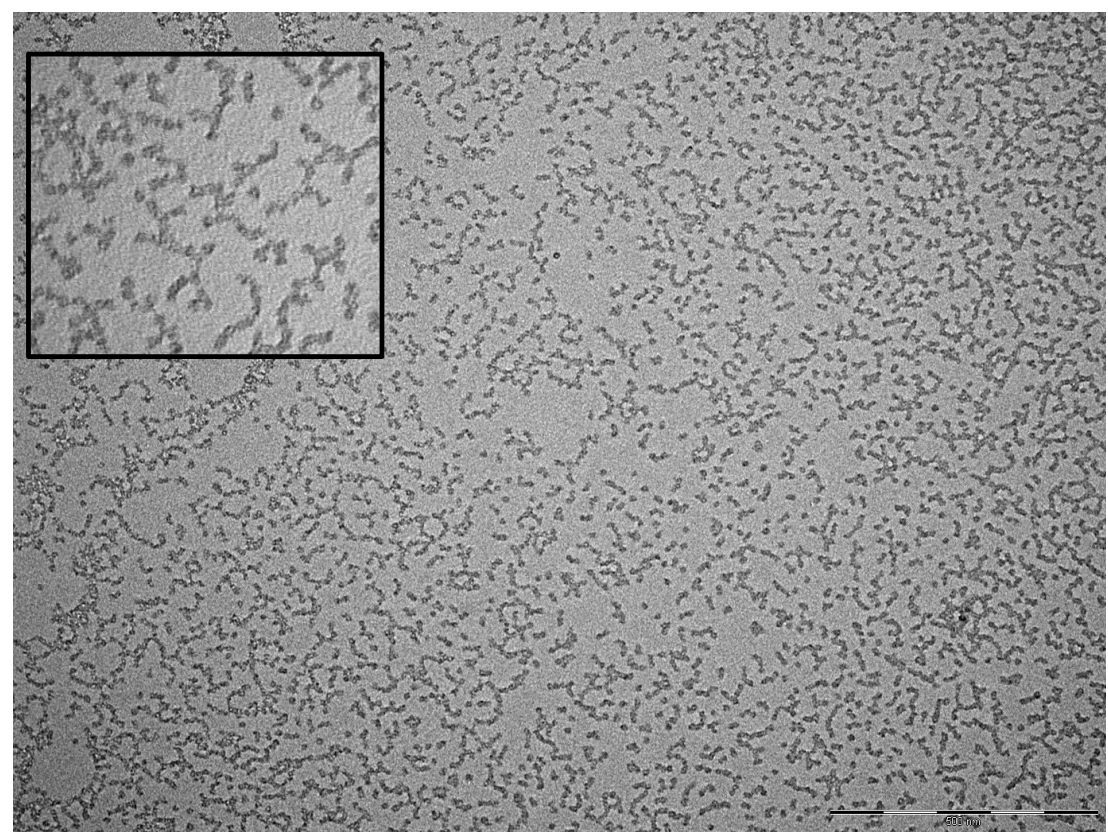

Figure S3. TEM images (magnifications in the inset) of PluS-NO NPs (scale bar $500 \mathrm{~nm}$ ). Only the dense silica core of diameter $=10 \mathrm{~nm} \pm 1$ can be seen with this technique. (Images taken with a Philips CM 100 TEM operating at $80 \mathrm{kV}$ ) 
TEMPO-CONHBU_H_CDCl3

TEMPO-CONHBu_H_CDCl3

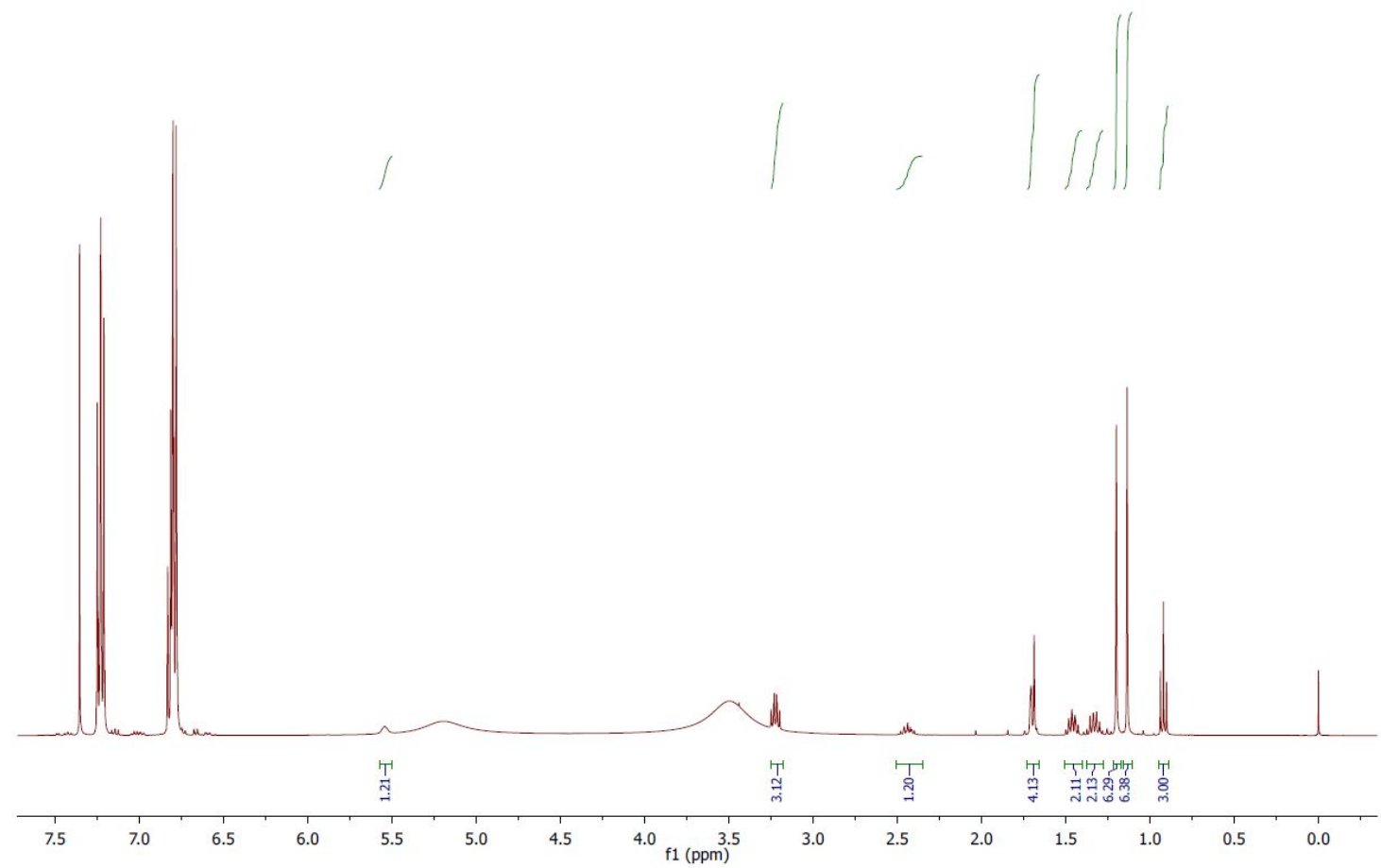

Figure S4. ${ }^{1} \mathrm{H}$ NMR spectrum of compound 6

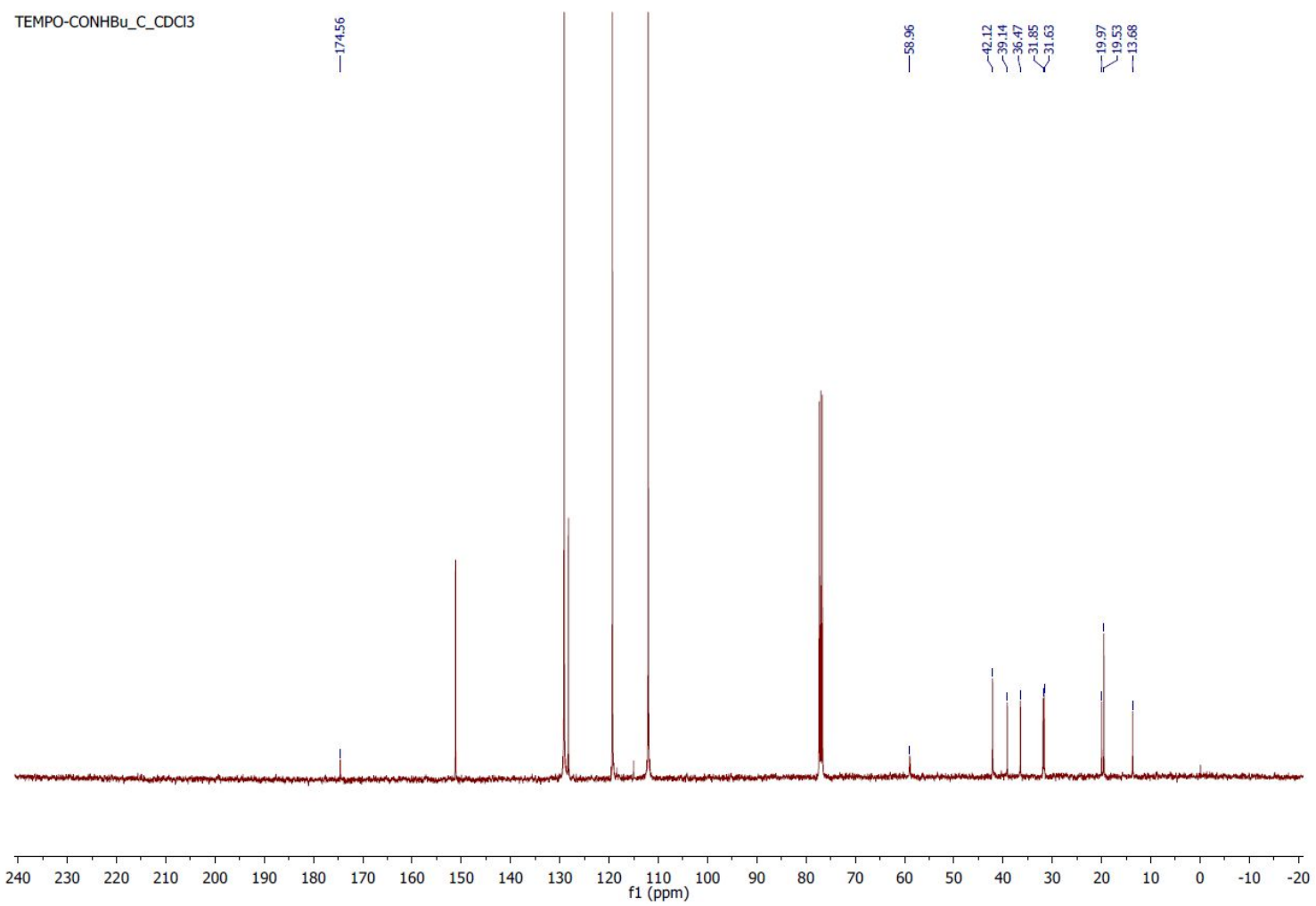

Figure $55 .{ }^{13} \mathrm{C}$ NMR spectrum of compound 6 


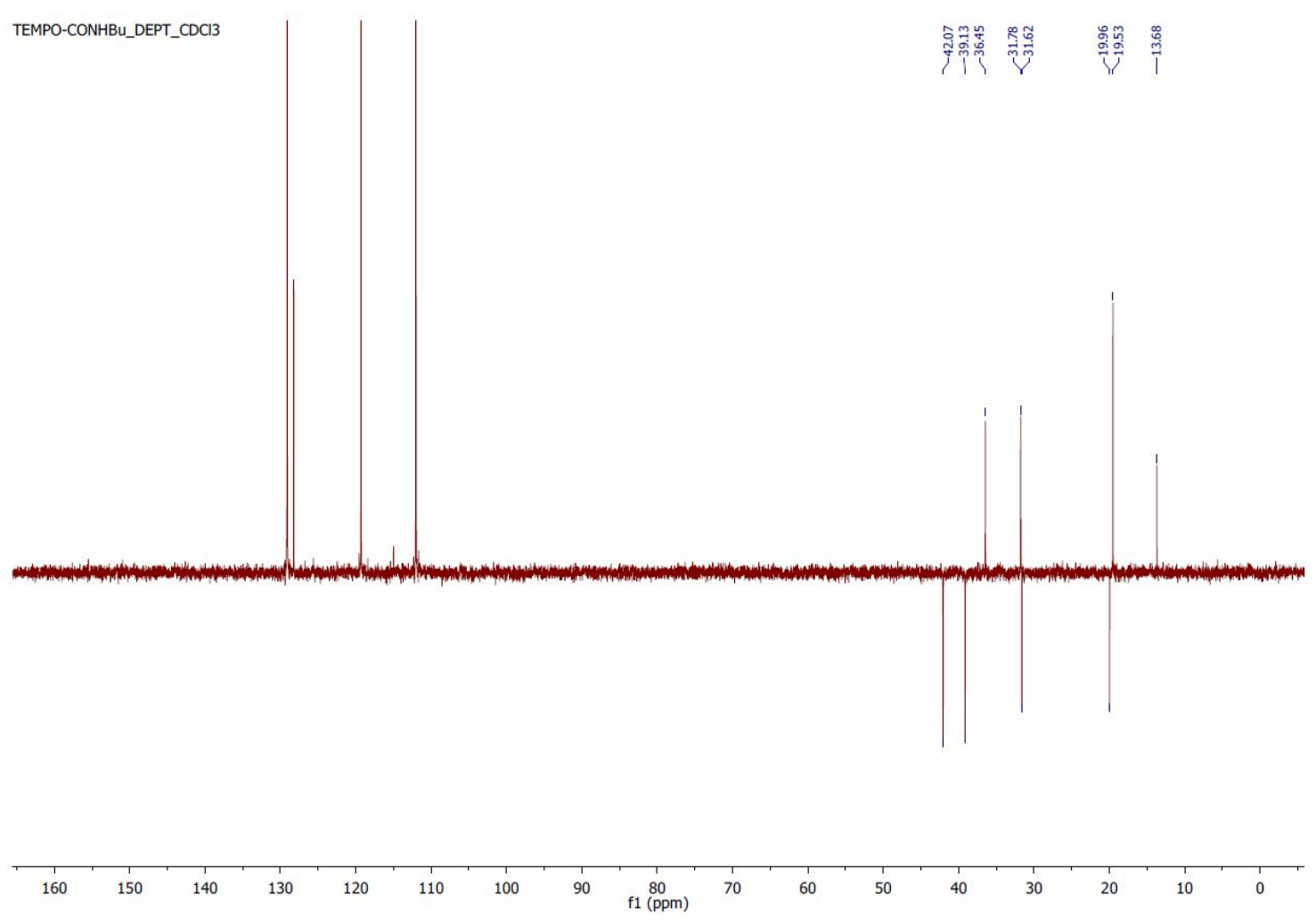

Figure S6. DEPT 135 spectrum of compound 6

FENILIDRAZINA_H_CDCl3

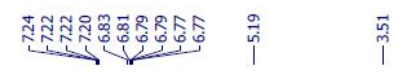

FENILIDRAZINA_C_CDCI3
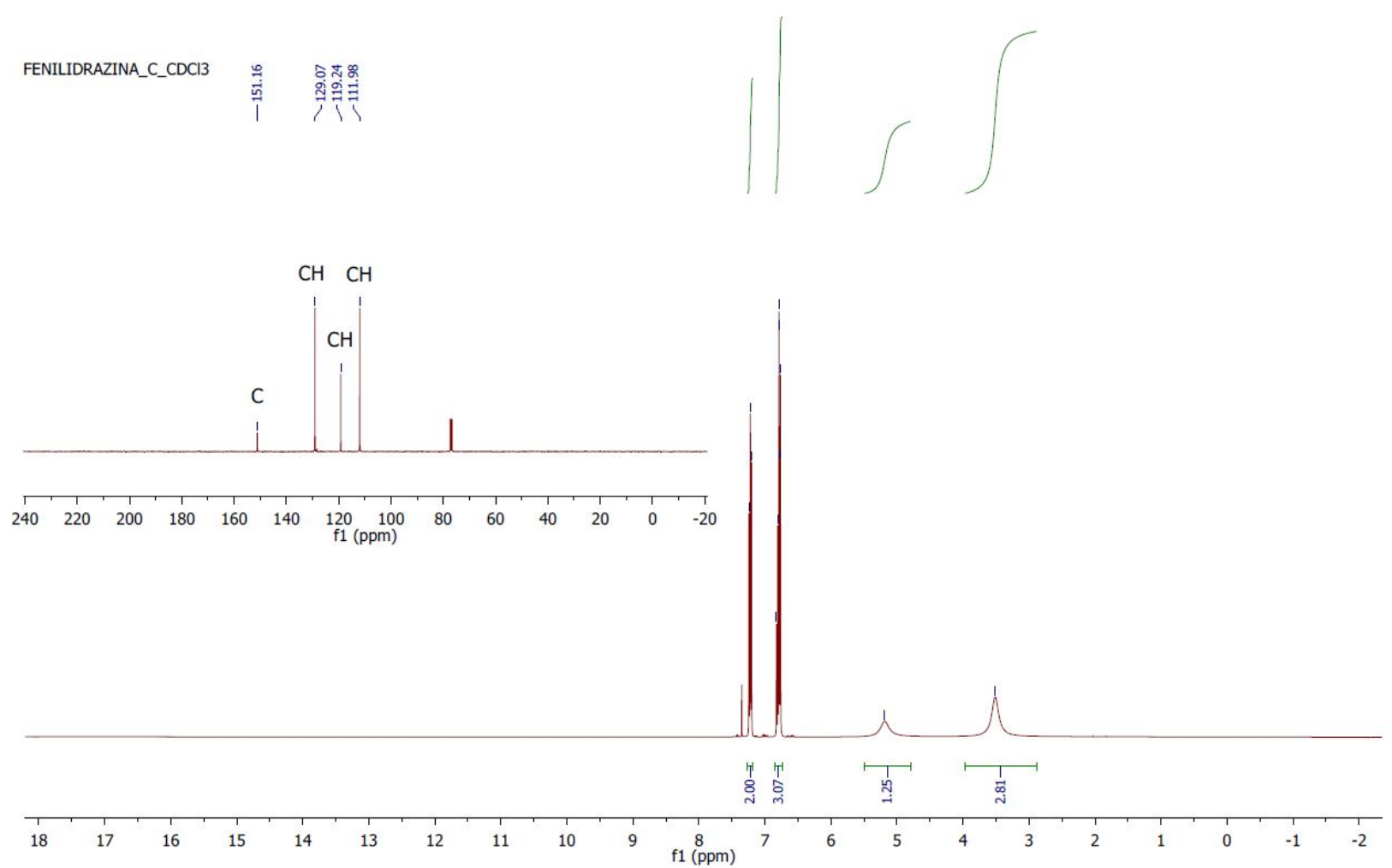

Figure S7. ${ }^{1} \mathrm{H}$ NMR and ${ }^{13} \mathrm{C}$ NMR spectrum of phenylhydrazine 


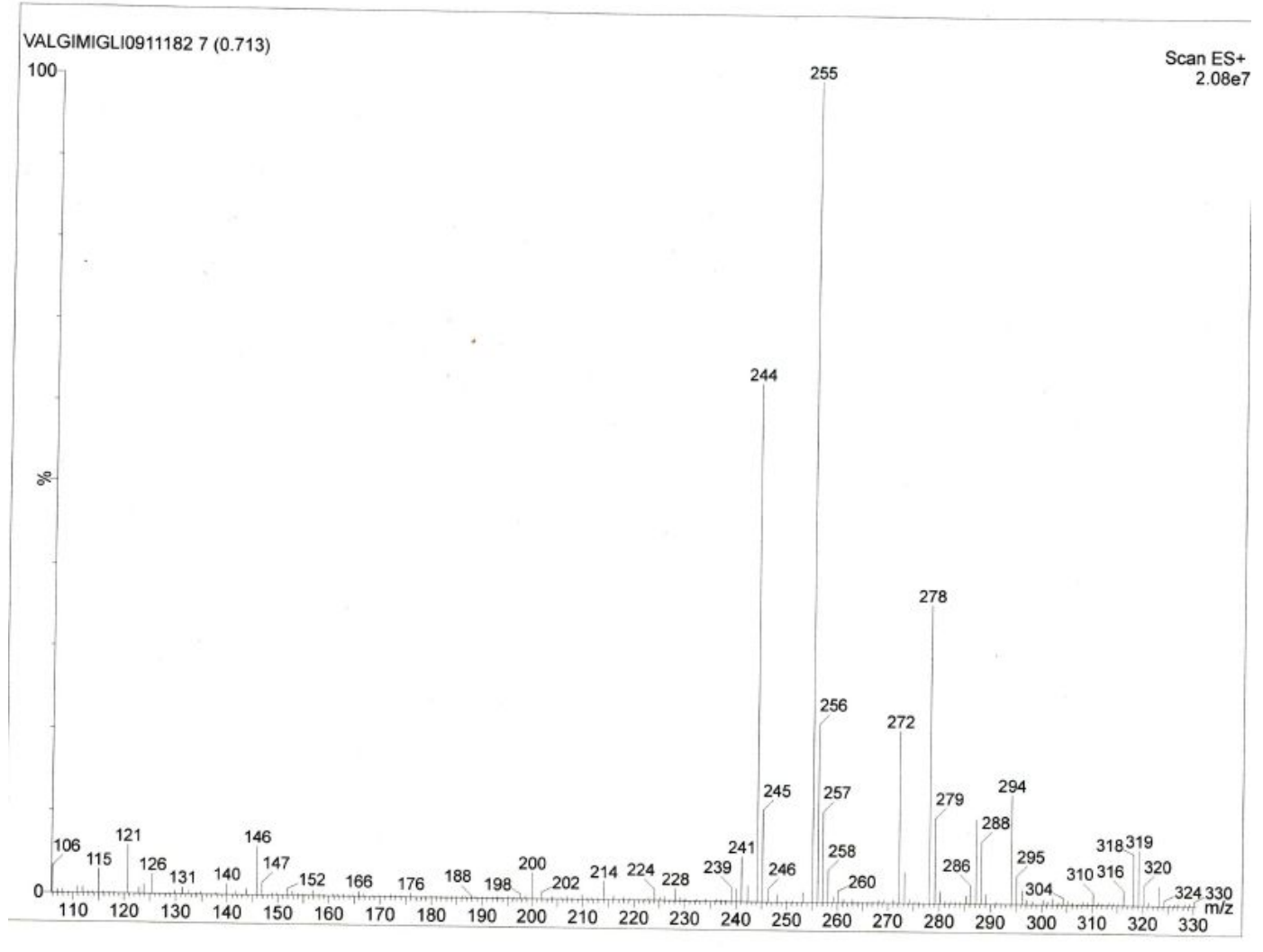

Figure S8. $\mathrm{ESI}^{+}$mass spectrum of compound 6 
1) Results of the numerical simulation of the experimental spectrum of Plus-NO (black).

The simulated spectrum contains two components. The first one has a low correlation time and an abundance of $92.6 \%$, and was identified as the bound nitroxide. The second one has a higher correlation time, an abundance of $7.4 \%$, and was interpreted as a trace of unreacted nitroxide adsorbed on the surface.

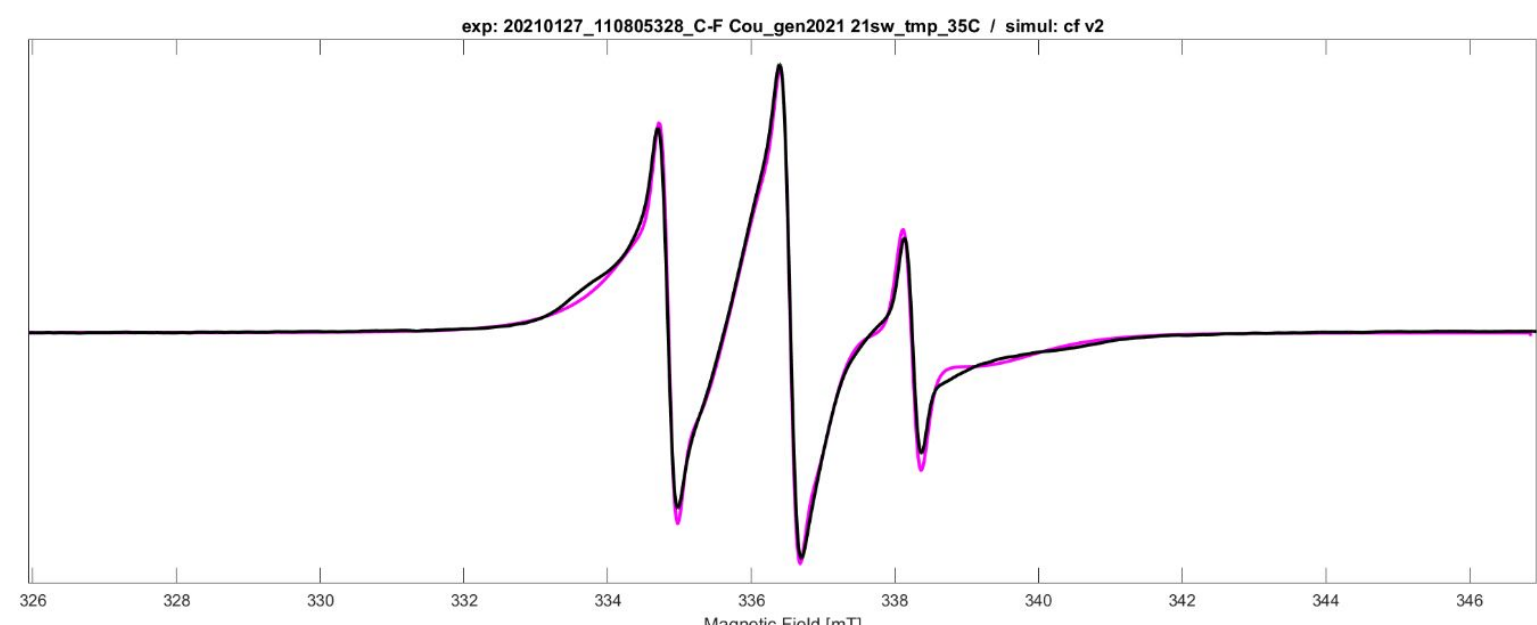

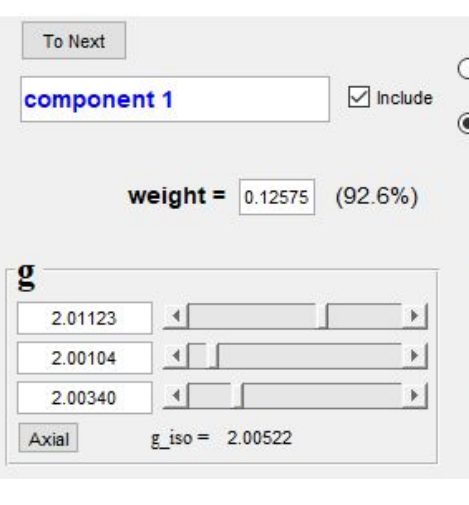

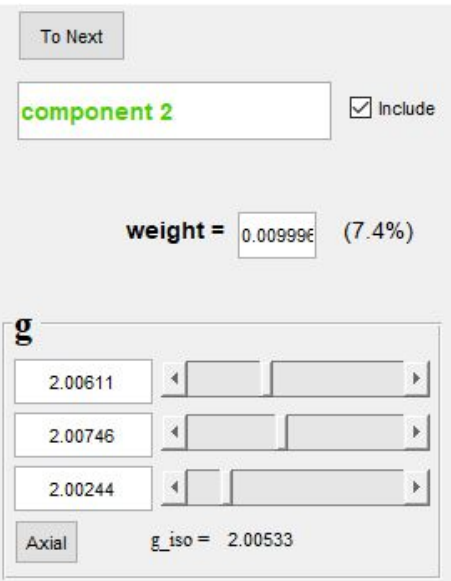

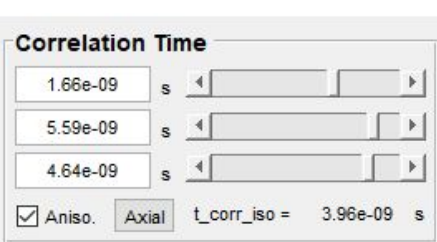
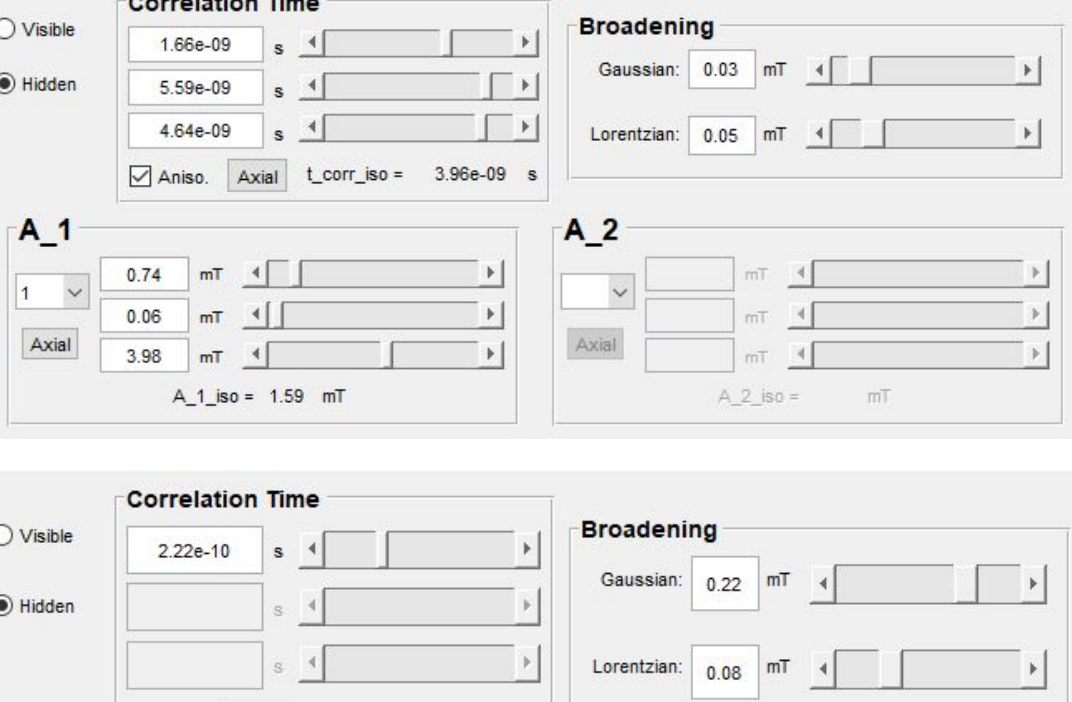

$\square$ Aniso. Axial t_corr_iso $=$

A_1

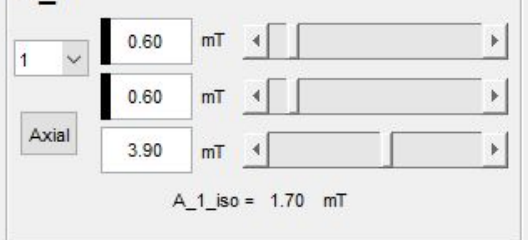

A_2

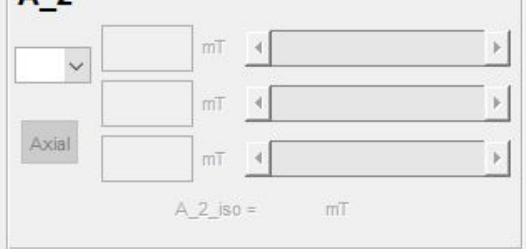


2) Results of the numerical simulation of the experimental spectrum of nitroxide 6 . The low correlation time is consistent with a dissolved nitroxide.
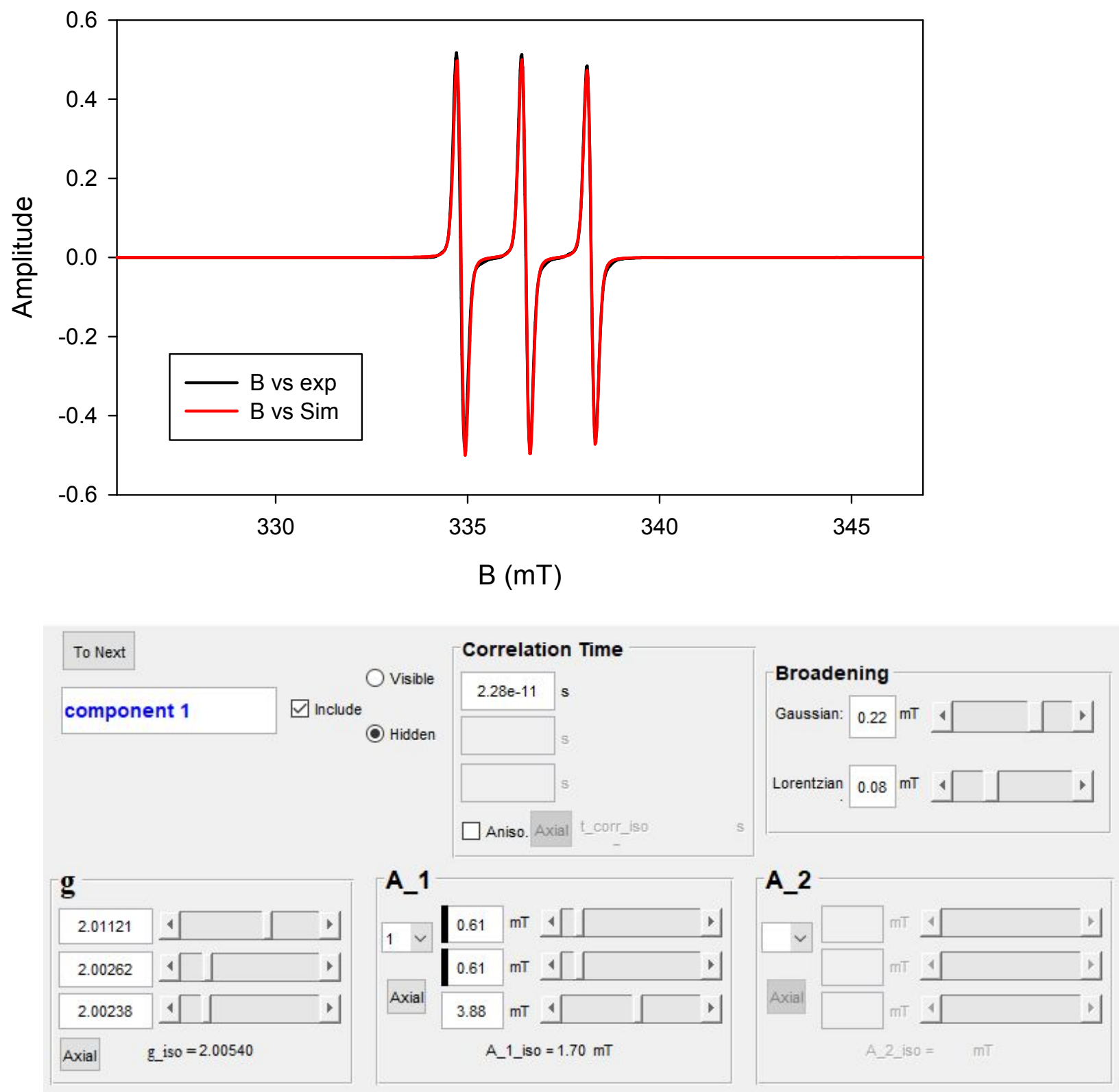
Numerical simulation of autoxidation of THF inhibited by nitroxides

Table S1. Reaction scheme and rate constants. $\mathrm{R} \bullet=$ alkyl radicals, $\mathrm{ROO} \bullet=$ peroxyl radicals, $\mathrm{RH}=\mathrm{THF}, \mathrm{NO} \bullet=$ nitroxide; $\mathrm{NOx}=$ oxoammonium cation, $\mathrm{NOH}=$ hydroxylamine.

\begin{tabular}{|l|l|l|}
\hline Reaction & Rate constant & note \\
\hline Initiator $\rightarrow \mathrm{R} \bullet$ & $\mathrm{Ri}=1.6 \mathrm{e}-9 \mathrm{Ms}^{-1}$ & Experimentally determined \\
\hline $\mathrm{R} \bullet+\mathrm{O}_{2} \rightarrow \mathrm{ROO} \bullet$ & $k=4 \mathrm{e} 9 \mathrm{M}^{-1} \mathrm{~s}^{-1}$ & Ref S2 \\
\hline $\mathrm{ROO} \bullet+\mathrm{RH} \rightarrow \mathrm{ROO}+\mathrm{R} \bullet$ & $k=4.8 \mathrm{M}^{-1} \mathrm{~s}^{-1}$ & Ref S3 \\
\hline $\mathrm{ROO} \bullet+\mathrm{ROO} \bullet \rightarrow$ Inactive Products & $k=3.3 e 7 \mathrm{M}^{-1} \mathrm{~s}^{-1}$ & Ref S3 \\
\hline $\mathrm{NO} \bullet+\mathrm{R} \bullet \rightarrow \mathrm{NOR}$ & fitted & \\
\hline $\mathrm{NO} \bullet+\mathrm{ROO} \bullet \rightarrow \mathrm{NOx}+\mathrm{ROOH}$ & Fitted & \\
\hline $\mathrm{NOx}+\mathrm{RH} \rightarrow \mathrm{NOH}+\mathrm{RHox}$ & Fitted & \\
\hline $\mathrm{NOH}+\mathrm{ROO} \bullet \rightarrow \mathrm{NO} \bullet+\mathrm{ROOH}$ & fitted & \\
\hline
\end{tabular}

Table S2. Results of numerical simulations.

\begin{tabular}{|l|l|l|l|l|}
\hline 4X-TEMPO & $k(\mathrm{NO} \bullet+\mathrm{ROO} \bullet) \mathrm{M}^{-1} \mathrm{~s}^{-1}$ & $k(\mathrm{NO} \bullet+\mathrm{R} \bullet) \mathrm{M}^{-1} \mathrm{~s}^{-1}$ & $k(\mathrm{NOx}+\mathrm{RH}) \mathrm{M}^{-1} \mathrm{~s}^{-1}$ & $k(\mathrm{NOH}+\mathrm{ROO} \bullet) \mathrm{M}^{-1} \mathrm{~s}^{-1}$ \\
\hline $\mathrm{H}(\mathbf{1})$ & $5.1 \mathrm{e} 6$ & $2.0 \mathrm{e} 11$ & $2 \mathrm{e}-3$ & $3.8 \mathrm{e} 6$ \\
\hline $\mathrm{OH} \mathrm{(2)}$ & $1.1 \mathrm{e} 6$ & $2.7 \mathrm{e} 10$ & 0.01 & $2.5 \mathrm{e} 6$ \\
\hline $\mathrm{NH}_{2}(\mathbf{3})$ & $5.4 \mathrm{e} 5$ & $1.5 \mathrm{e} 10$ & 0.02 & $1.0 \mathrm{e} 6$ \\
\hline $\mathrm{COOH}(\mathbf{4})$ & $3.7 \mathrm{e} 5$ & $4.0 \mathrm{e} 10$ & $6 \mathrm{e}-4$ & $4.3 \mathrm{e} 6$ \\
\hline $\mathrm{NHCOCH}_{3}(\mathbf{5})$ & $1.1 \mathrm{e} 5$ & $2.6 \mathrm{e} 10$ & 0.01 & $2.3 \mathrm{e} 6$ \\
\hline $\mathrm{CONHBut} \mathrm{(6)}$ & $1.9 \mathrm{e} 5$ & $2.7 \mathrm{e} 10$ & $5 \mathrm{e}-4$ & $4.5 \mathrm{e} 6$ \\
\hline$=\mathrm{O}(\mathbf{7})$ & $5.6 \mathrm{e} 4$ & $1.9 \mathrm{e} 10$ & $3.6 \mathrm{e}-3$ & $2.0 \mathrm{e} 6$ \\
\hline PLUS-NO & $1.5 \mathrm{e} 5$ & $1.8 \mathrm{e} 10$ & $2.4 \mathrm{e}-4$ & $1.2 \mathrm{e} 6$ \\
\hline
\end{tabular}


nitroxides

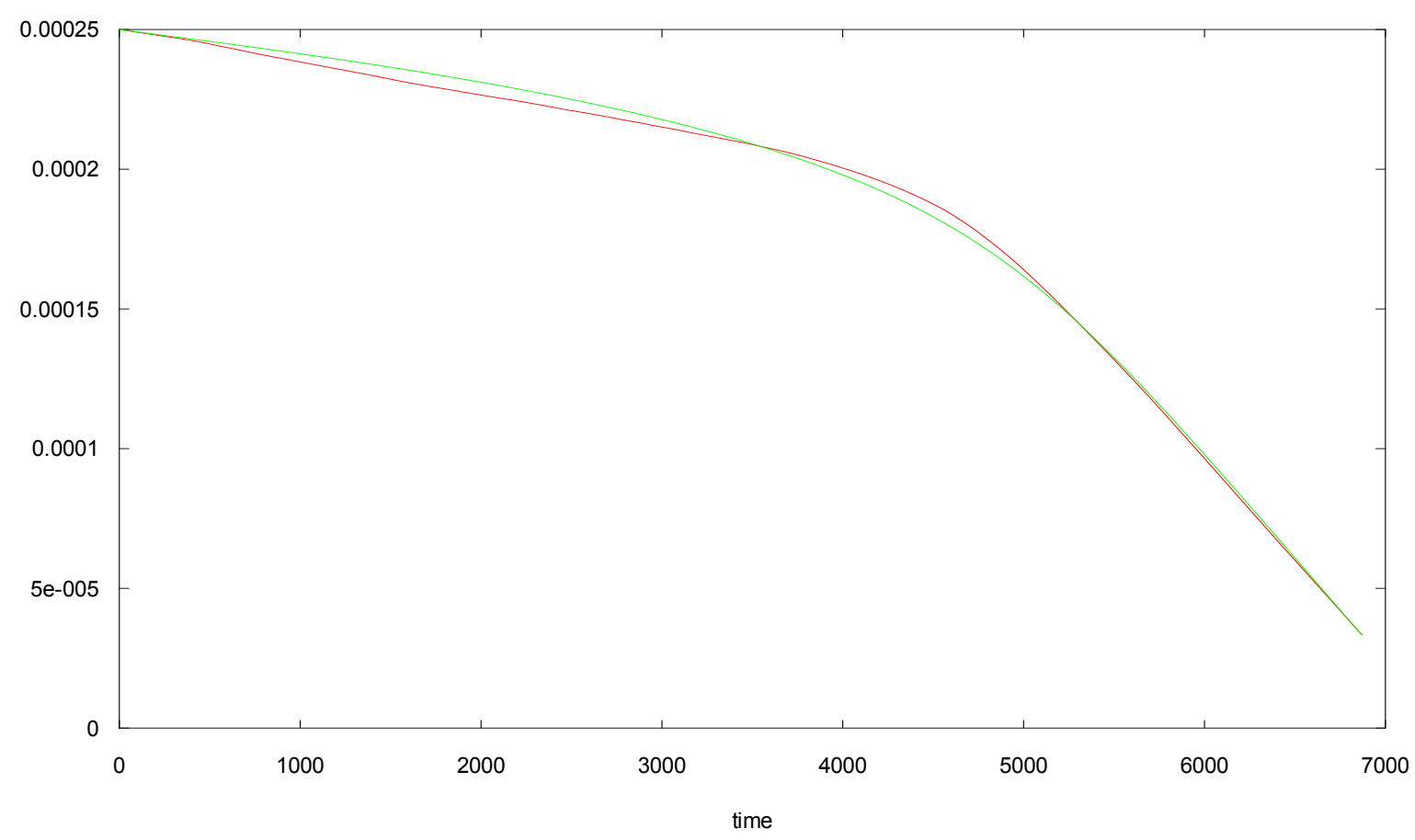

Figure S9. Example of result obtained by the numerical simulation. Autoxidation of THF inhibited by hydroxyl TEMPO (2), experimental trace (red) and simulated trace (green). $\mathrm{Y}$-axis: $\left[\mathrm{O}_{2}\right]$.

\section{References}

S1 Cicco S. R.; Vona D.; De Giglio E.; Cometa S.; Mattioli-Belmonte M.; Palumbo F.; Ragni R.; Farinola G. M. Chemically Modified Diatoms Biosilica for Bone Cell Growth with Combined Drug-Delivery and Antioxidant Properties. Chem. Plus. Chem 2015, 80, $1104-1112$.

S2 Maillard B.; Ingold K. U.; Scaiano J. C. Rate constants for the reactions of free radicals with oxygen in solution. J. Am. Chem. Soc. 1983, 105, 5095-5099

S3 Amorati R.; Baschieri A.; Morroni G.; Gambino R.; Valgimigli L. Peroxyl Radical Reactions in Water Solution: A Gym for Proton-Coupled Electron-Transfer Theories. Chem. Eur. J. 2016, 22, $7924-7934$ 